\title{
Increased carotid artery intima-media thickness and myeloperoxidase level in children with newly diagnosed juvenile idiopathic arthritis
}

Jaanika llisson ${ }^{1,2^{*}}$, Maksim Zagura ${ }^{3,4}$, Kersti Zilmer ${ }^{3}$, Erik Salum ${ }^{4}$, Kaire Heilman ${ }^{1,5}$, Anneli Piir ${ }^{3}$, Vallo Tillmann ${ }^{1,2}$, Jaak Kals ${ }^{3,6}$, Mihkel Zilmer $^{3}$ and Chris Pruunsild ${ }^{1,2}$

\begin{abstract}
Introduction: Juvenile idiopathic arthritis (JIA) is a frequent childhood rheumatic disease characterized by chronic inflammation. The latter has been related to impairment of arterial functional-structural properties, atherogenesis and later cardiovascular events. The objective of this study was to examine intima-media thickness (IMT) and the parameters of arterial stiffness in children with JIA at diagnosis and their correlation with JIA subtype and markers of inflammation and atherosclerosis.
\end{abstract}

Methods: Thirty-nine newly diagnosed patients with JIA (26 girls; mean age, $13.2 \pm 2.6$ years) and 27 healthy controls (9 girls; mean age, 13.6 3.4 years) were included in the study. Twelve patients had oligoarthritis, fifteen had extended oligoarthritis and twelve had rheumatoid factor-negative polyarthritis. IMT of the common carotid artery was determined by ultrasonography, carotid-femoral pulse wave velocity (cfPWV) and augmentation index adjusted to a heart rate of 75 beats/min (Alx@75) were determined by applanation tonometry. The serum levels of atherosclerosis-related biomarkers, such as asymmetric dimethylarginine (ADMA), myeloperoxidase (MPO) and adiponectin, were measured by enzyme-linked immunosorbent assay.

Results: Mean IMT ( $0.46 \pm 0.04$ vs. $0.42 \pm 0.04 \mathrm{~mm} ; p=0.0003)$ and MPO concentration (115.2 [95 \% confidence interval $\{95 \% \mathrm{Cl}\}, 97.4-136.3]$ vs. $57.6[95 \% \mathrm{Cl}, 47.1-70.3] \mathrm{ng} / \mathrm{ml} ; p<0.0001)$ were higher in the patients with JIA than in the control subjects. The cfPWV, Alx@75 and serum ADMA and adiponectin levels did not significantly differ between the groups and JIA subtypes. Serum adiponectin level correlated negatively with Alx@75 in patients with JIA $(r=-0.38 ; p<0.05)$.

Conclusions: Patients with JIA have increased mean IMT and elevated MPO levels at early stages of the disease. Alx@75 was inversely independently associated with adiponectin level in the patients, suggesting that lower adiponectin levels might influence arterial subclinical stiffening in patients with newly diagnosed JIA.

\section{Introduction}

Juvenile idiopathic arthritis (JIA) is the most common chronic rheumatic disease in childhood, with an onset before the age of 16 years and a potential to persist into adulthood. JIA is a heterogeneous group of disorders with different disease progression and prognosis. Of the JIA subtypes, persistent oligoarthritis and monocyclic systemic arthritis have the best prognosis and polyarthritis

\footnotetext{
* Correspondence: jaanika.ilisson@kliinikum.ee

'Department of Paediatrics, Faculty of Medicine, University of Tartu, Lunini 6, Tartu 51014, Estonia

${ }^{2}$ Children's Clinic, Tartu University Hospital, Tartu, Estonia

Full list of author information is available at the end of the article
}

the worst $[1,2]$. Approximately $50 \%$ of patients with JIA still have active disease and need for disease-modifying antirheumatic medications in adulthood [3-5].

Many patients with active JIA may not present with high levels of classical inflammatory markers such as C-reactive protein or erythrocyte sedimentation rate. Therefore, studies on the role of new markers (e.g., myeloperoxidase [MPO], asymmetric dimethylarginine [ADMA], adiponectin) may open some new aspects to describe inflammation in patients with JIA. MPO, ADMA and adiponectin have been linked to inflammation and/or oxidative stress [6-8]. Previous studies have shown that MPO levels are higher in patients with JIA compared with control groups [9]. 
ADMA and adiponectin have not been investigated in patients with JIA.

Atherosclerosis is considered an inflammatory disorder starting in childhood. Chronic inflammation has been suggested to play an important role in the developing of early atherosclerosis, which contributes to the development of cardiovascular disease (CVD) [10, 11].

CVD is more frequent in patients with rheumatoid arthritis (RA), and they are diagnosed at an earlier age than other patients with CVD. Mortality among RA patients is also higher than general population and is mostly due to cardiovascular diseases [12-14]. In addition to traditional risk factors for CVD, patients with a chronic inflammatory disease also have many disease-specific and drug-related risk factors [13]. Recent studies have shown that patients with RA have increased aortic stiffness, and this is correlated with markers of inflammation and aortic inflammation without any clinical signs of CVD $[15,16]$.

As JIA is a chronic inflammatory disease, it has longterm effects on many organ systems, including the cardiovascular system. Accordingly, it has been shown that patients with JIA have a moderate risk for premature cardiovascular events [17].

To date, only a few studies of vascular function, structural and atherosclerosis-related biomarkers in patients with JIA have been done. They have shown impaired endothelial dysfunction and alterations in vascular function compared with controls during the course of the disease $[18,19]$. Satija et al. reported altered arterial wall indices in newly diagnosed patients with JIA, indicating increased arterial stiffness [20].

The aim of the present study was to evaluate intimamedia thickness (IMT) and the parameters of arterial stiffness in patients with JIA at the time of diagnosis and to assess their correlation with JIA subtype and serum markers of inflammation and atherosclerosis.

\section{Methods}

\section{Patients and controls}

This cross-sectional study included 39 children with JIA (26 girls; mean age, $13.2 \pm 2.6$ years) who were patients of the paediatric rheumatologist at the outpatient children's clinic of Tartu University Hospital, Estonia, and 27 healthy control subjects (9 girls; mean age, $13.6 \pm$ 3.4 years) recruited from a previous diabetes study [21]. The study group included children aged 7-18 years with newly diagnosed JIA (onset of symptoms before the 16th birthday). JIA was diagnosed according to the International League of Associations for Rheumatology criteria [22]. Twenty-seven patients had oligoarthritis (fifteen of them had extended oligoarthritis), and twelve had rheumatoid factor (RF)-negative polyarthritis. All patients were RF-negative, three were antinuclear antibody-positive and five were human leukocyte antigen B27-positive.

Healthy control subjects were defined as without known chronical illness at the time of enrolment, without acute infection and no history of using antihypertensive or lipid-lowering medications. They were matched with the patients by age ( \pm 2 years). Three of the subjects from the original control group were excluded because of greater age difference. Written informed consent was obtained from each participant and the parent or legal representative. The study was approved by the ethics committee of Tartu University.

\section{Clinical and laboratory investigations}

A full joint examination was performed by the same paediatric rheumatologist during the outpatient visit. Blood samples were obtained for nine different biochemical markers: glucose, creatinine, total cholesterol, high-density lipoprotein (HDL) cholesterol, lowdensity lipoprotein (LDL) cholesterol, triglycerides, ADMA, adiponectin and MPO. The first six markers are regularly used in clinical practice and were analysed in the local clinical laboratory using certified standard assays. For the last three markers, serum and plasma were stored at $-80{ }^{\circ} \mathrm{C}$ until analysis. Serum ADMA concentration was determined by using a validated enzyme-linked immunosorbent assay (ELISA) kit (DLD Gesellschaft für Diagnostika und medizinische Geräte $\mathrm{mbH}$, Hamburg, Germany). Serum adiponectin concentration was determined using the Human Adiponectin/Acrp30 Quantikine ELISA kit (R\&D Systems Europe, Abingdon, UK). The adiponectin intraassay coefficient of variation $(\mathrm{CV})$ is $4.6 \%$, and the interassay $\mathrm{CV}$ is $7.9 \%$. MPO was determined in serum of patients with JIA using an enzyme immunoassay test kit (catalogue number BC-1129; BioCheck, Foster City, CA, USA) and in plasma of controls using an ELISA kit (BIOXYTECH enzyme immunoassay for MPO, catalogue number 21013; OXIS Health Products, Portland, OR, USA), according to the manufacturer's instructions. MPO intraassay CV is $5.7 \%$ and inter-assay CV $8.5 \%$.

\section{Haemodynamic measurements}

Brachial blood pressure (BP) and heart rate were recorded from the left arm with the subject in the supine position. BP was measured with an automated digital oscillometric BP monitor (OMRON M4-I; Omron Healthcare Europe, Hoofddorp, the Netherlands). Radial arterial pressure waveforms were obtained with a high-fidelity applanation tonometer (SPT-301B; Millar, Houston, TX, USA) applied to the wrist of the left hand. Pulse wave analysis (SCOR-Px 7.0; AtCor Medical, West Ryde, Australia) was then performed to generate a corresponding central (ascending aortic) 
waveform using a generalized transfer function, which has been prospectively validated for assessment of ascending aortic BP [23]. Augmentation index (AIx), mean arterial pressure (MAP), central systolic blood pressure, central diastolic blood pressure and central pulse pressure were determined by pulse wave analysis. The AIx was calculated as the difference between the second and the first systolic peaks, divided by pulse pressure and expressed in percentages [24]. The AIx values were adjusted to a heart rate of 75 beats/ min (AIx@75) using a built-in algorithm in the SphygmoCor Px system (AtCor Medical). The within- and between-observer CVs for AIx@75 were 3.4 \% and 7.1 \%, respectively. Pulse wave velocity was measured by the foot-to-foot method using the same device. The aortic pulse wave velocity (aPWV) was determined by sequentially recording electrocardiogram-gated carotid and femoral artery waveforms, as described in detail previously [24]. The within- and between-observer CVs for aPWV were $2.3 \%$ and $6.2 \%$, respectively. All measurements were made in duplicate by two trained investigators, and the mean values were used in subsequent analysis.

\section{Assessment of carotid intima-media thickness}

Carotid ultrasound for evaluation of IMT was performed using the SonoSite M-Turbo portable ultrasound device (SonoSite, Bothell, WA, USA) coupled to a 5- to $10-\mathrm{MHz}$ multifrequency high-resolution linear transducer. SonoCalc software (SonoSite) was used for calculation of mean IMT values. The measurements were obtained with the subject lying down, with the head extended and slightly turned opposite to the examined carotid. Measurements were made of the common carotid artery after the examination of a longitudinal section of $10 \mathrm{~mm}$ at a distance of $1 \mathrm{~cm}$ from the bifurcation. The measurements were performed in the far wall in the lateral, anterior and posterior projections. The within- and between-observer CVs for mean IMT were $2.2 \%$ and $5.9 \%$, respectively.

A standard deviation score (SDS) of IMT for each subject was calculated using height-specific IMT normative values published by Jourdan et al. [25].

\section{Statistical analysis}

Differences between the JIA and control groups were studied with analysis of variance, adjusted by sex. Because the distributions of some variables were not normally distributed, they were log-transformed for analysis as required to improve normality. The normally distributed data are presented as mean \pm standard deviation; the non-normally distributed data are presented as the geometric mean with the $95 \%$ confidence interval (CI). Multiple regression analysis was performed to investigate the independent determinants of AIx@75. To examine the associations between the variables, Pearson's correlation test was used.

We used AIx@75 as a base for power calculations for $t$ tests. A total of 66 persons were targeted to enter this two-group study. The probability is $80 \%$ that the study will detect an effect size (the smallest difference between the means) at a two-sided 0.05 significance level if the true difference between groups is $4.953 \mathrm{U}$. This is based on the assumption that the standard deviation of the response variable is 10 [26]. Differences were considered statistically different if the $p$ value was less than 0.05. Statistical analysis was performed using the SAS version 9.2 statistical software package (SAS Institute, Cary, NC, USA).

\section{Results}

The subjects' clinical and laboratory characteristics, as well as the arterial functional-structural properties of the study groups, are presented in Table 1 .

The total cholesterol, LDL cholesterol, HDL cholesterol and triglyceride levels did not differ between groups. The same was found for serum ADMA and adiponectin levels (Table 1).

The serum level of MPO was significantly higher in patients with JIA compared with the plasma MPO concentrations in control subjects (115.2 [95\% CI, 97.4-136.3] vs. 57.6 [95 \% CI, $47.1-70.3] \mathrm{ng} / \mathrm{ml} ; p<0.0001$ ).

Mean IMT values and IMT height specific SDS were higher in the patients with JIA than in the control subjects $(0.46 \pm 0.04$ vs. $0.42 \pm 0.04 \mathrm{~mm}, p<0.0003 ; 1.694 \pm$ 0.858 vs. $0.744 \pm 0.852, p=0.0002)$. We did not find statistically significant differences between the groups with regard to carotid-femoral pulse wave velocity (cfPWV) or AIx@75 (Table 1).

Serum ADMA and adiponectin levels, as well as the mean IMT, cfPWV and AIx@75, did not differ significantly between the JIA subtypes (data not shown).

There was a significant negative correlation between serum adiponectin level and AIx@75 in patients with JIA $(r=-0.38 ; p<0.05)$ (Fig. 1$)$. No other statistically significant correlations were found.

In multiple regression analysis, AIx@75 was independently determined by age, MAP and adiponectin levels in patients with JIA, accounting for $29 \%$ of its variability $\left(R^{2}(\operatorname{adj})=0.29 ; p<0.01\right)($ Table 2$)$.

\section{Discussion}

We have demonstrated that, already at the time of JIA diagnosis, there are atherosclerosis-related changes in the arterial wall, supporting the detrimental effect of systemic inflammation on the cardiovascular system. The present study is the first clinical analysis of the relationship between adiponectin and arterial stiffness in patients with JIA. We have shown 
Table 1 Clinical and laboratory data and arterial functional-structural properties in children with juvenile idiopathic arthritis and controls, adjusted by sex

\begin{tabular}{|c|c|c|c|}
\hline Parameters & JIA group $(n=39)$ & Control group $(n=27)$ & $p$ value \\
\hline Age (yr) & $13.0 \pm 3.11$ & $13.5 \pm 3.08$ & 0.5687 \\
\hline Body mass index $\left(\mathrm{kg} / \mathrm{m}^{2}\right)$ & $21.2 \pm 4.09$ & $20.2 \pm 4.04$ & 0.3364 \\
\hline Peripheral systolic blood pressure $(\mathrm{mmHg})$ & $112.2 \pm 8.7$ & $108.9 \pm 8.6$ & 0.1375 \\
\hline Peripheral diastolic blood pressure (mmHg) & $57.6 \pm 5.01$ & $57.1 \pm 4.96$ & 0.7360 \\
\hline Mean arterial blood pressure $(\mathrm{mmHg})$ & $74.0 \pm 5.82$ & $73.3 \pm 5.77$ & 0.6549 \\
\hline Central systolic blood pressure $(\mathrm{mmHg})$ & $92.0 \pm 7.5$ & $90.17 \pm 7.4$ & 0.3748 \\
\hline Central diastolic blood pressure $(\mathrm{mmHg})$ & $58.5 \pm 5.07$ & $58.4 \pm 5.04$ & 0.9249 \\
\hline Cholesterol $(\mathrm{mmol} / \mathrm{L})^{a}$ & $4.1(3.8-4.3)$ & $4.1(3.8-4.4)$ & 0.8150 \\
\hline LDL cholesterol $(\mathrm{mmol} / \mathrm{L})^{a}$ & $2.24(2.06-2.43)$ & $2.36(2.13-2.60)$ & 0.4324 \\
\hline $\mathrm{HDL}$ cholesterol (mmol/L) & $1.50 \pm 0.40$ & $1.71 \pm 0.40$ & 0.0415 \\
\hline Triglycerides $(\mathrm{mmol} / \mathrm{L})^{\mathrm{a}}$ & $0.87(0.75-1.02)$ & $0.70(0.59-0.84)$ & 0.0688 \\
\hline ADMA $(\mu \mathrm{mol} / \mathrm{L})$ & $0.622 \pm 0.175$ & $0.679 \pm 0.173$ & 0.2040 \\
\hline MPO $(\mathrm{ng} / \mathrm{ml})^{\mathrm{a}}$ & $115.2(97.4-136.3)$ & $57.6(47.1-70.3)$ & $<0.0001$ \\
\hline Adiponectin $(\mathrm{ng} / \mathrm{ml})^{\mathrm{a}}$ & $8596.7(7296.3-10,128.9)$ & $9123.1(7506.6-11,087.7)$ & 0.6519 \\
\hline Mean IMT (mm) & $0.46 \pm 0.04$ & $0.42 \pm 0.04$ & 0.0003 \\
\hline IMT SDS, height-specific ${ }^{b}$ & $1.694 \pm 0.858$ & $0.744 \pm 0.852$ & 0.0002 \\
\hline $\mathrm{cfPWV}(\mathrm{m} / \mathrm{s})^{\mathrm{a}}$ & $4.92(4.71-5.14)$ & $5.18(4.90-5.47)$ & 0.1579 \\
\hline Alx@75 (\%) & $0.642 \pm 11.258$ & $-2.042 \pm 11.179$ & 0.3811 \\
\hline
\end{tabular}

ADMA Asymmetric dimethylarginine, Alx@75 augmentation index values adjusted to a heart rate of 75 beats/min, cfPWV carotid-femoral pulse wave velocity, $H D L$ high-density lipoprotein cholesterol, JIA juvenile idiopathic arthritis, LDL low-density lipoprotein cholesterol, MPO myeloperoxidase, SDS standard deviation score Values are mean \pm standard deviation unless otherwise indicated

${ }^{\mathrm{a} D a t a}$ were log-transformed before statistical analysis and are presented as geometric mean (95\% confidence interval)

${ }^{\mathrm{b}}$ Data for34 patents with JIA and 23 control were analysed as reference values and are available for the height of $140 \mathrm{~cm}$ and taller [25]

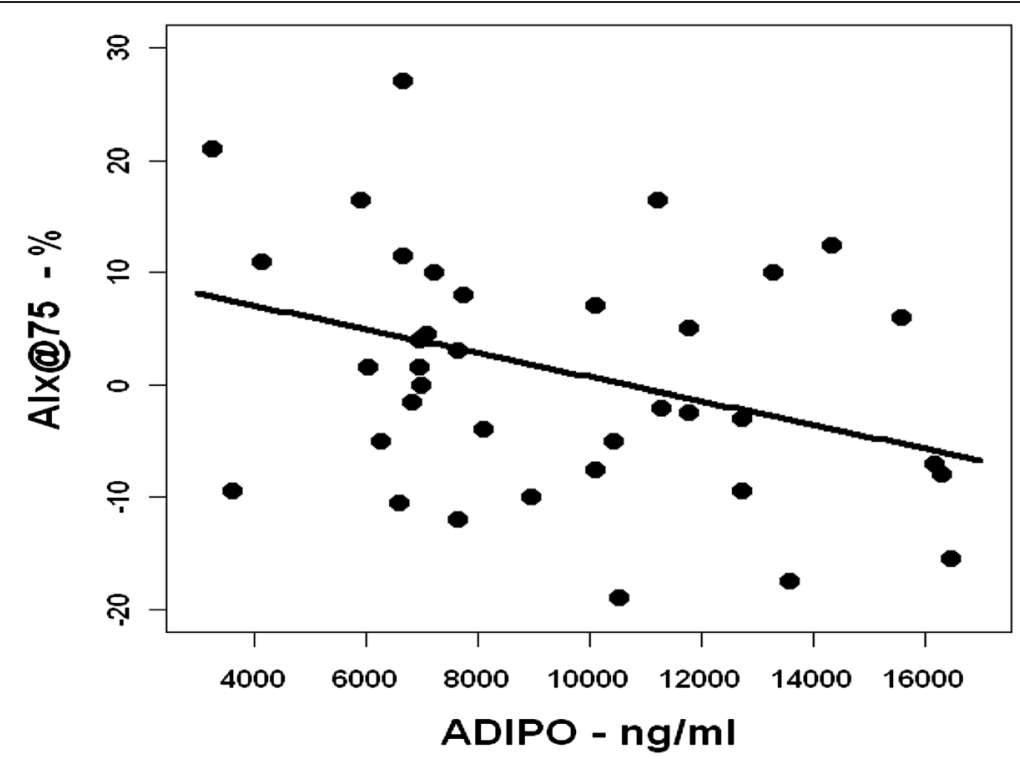

Fig. 1 Correlation between serum adiponectin (ADIPO) concentration and augmentation index values adjusted to a heart rate of 75 beats/min (Alx@75) in patients with juvenile idiopathic arthritis $(r=-0.38 ; p<0.05)$ 
Table 2 Multiple regression model for patients with juvenile idiopathic arthritis with augmentation index values adjusted to a heart rate of 75 beats/min as the dependent variable

\begin{tabular}{llll}
\hline Parameter & Regression coefficient & Standard deviation & $p$ value \\
\hline Age & -1.71 & 0.62 & 0.009 \\
MAP & 0.73 & 0.27 & 0.01 \\
Adiponectin & -0.001 & 0.0004 & 0.03 \\
\hline
\end{tabular}

MAP mean arterial pressure

that AIx@75 is inversely related to adiponectin levels in a cohort of patients with JIA. This finding suggests that adiponectin might influence arterial stiffening in the early stages of the disease. In addition, we demonstrated that serum MPO is significantly higher in patients with JIA. Thus, MPO may be a sensitive marker of systemic inflammation and oxidative stress in patients with newly diagnosed JIA.

To date, IMT in patients with JIA has been reported in a few studies, with controversial results. Breda et al. reported increased mean IMT in these patients [19], whereas Vlahos et al. did not find a statistically significant difference in IMT between their JIA and control groups [18]. Comparing mean IMT between JIA subtypes, Vlahos et al. found that a systemic arthritis (high inflammatory activity) group had a thicker intima-media layer compared with the control group and the oligoarticular group [18]. Both of these studies were done in patients with previously diagnosed JIA $[18,19]$. By contrast, our study was conducted with patients at the very early stage of the disease, and we demonstrated that IMT is increased in patients with JIA already in the early phase. We did not find a significant difference in IMT thickness between the oligo- and polyarticular subtypes. So far, only Breda et al. have investigated the changes in IMT longitudinally in patients with JIA. They found that after a 12-month treatment period, the mean IMT improved; that is, was less than at the start of the study [19]. This points out the importance of early, adequate, aggressive treatment of JIA to controlling the possibility of future cardiovascular events. Future investigations are needed to verify this point.

Several studies have shown that increased MPO concentration is associated with an increased risk of CVD $[27,28]$. Also, studies have shown higher serum and plasma concentrations in patients with rheumatologic diseases $[9,29]$. In our study, we found higher serum levels of MPO in patients with JIA already at an early stage of the disease. Thus, MPO may be a good marker of the level of inflammation, even when the classical inflammation markers are at normal levels.

ADMA acts as a natural inhibitor of nitric oxide synthase by reducing nitric oxide production. Higher levels of ADMA give rise to endothelial dysfunction and arterial stiffening [30]. The key enzyme in degradation of
ADMA is dimethylarginine dimethylaminohydrolase, and its activity is downregulated by tumour necrosis factor $\alpha$ and has a known role in the pathogenesis of RA and JIA [31]. Higher levels of ADMA and its correlation to oxidative stress markers have been shown in patients with RA [7]. In children with other chronic diseases, such as chronic kidney disease or type 1 diabetes mellitus, elevated serum ADMA concentrations have been found [21, 32]. However, all these children already had their disease for many years. To our knowledge, the level of ADMA has not been investigated to date in patients with JIA. In our study, we did not find a significant difference in serum ADMA levels at the disease start between our patient and control groups.

Adiponectin has well-known anti-inflammatory properties. To the contrary, there are studies suggesting that hypoadiponectemia might play an important role in CVDs (e.g., hypertension) [33]. In patients with RA, adiponectin levels are higher than in patients with noninflammatory conditions (e.g., osteoarthrosis), which is suggestive of a proinflammatory effect [8]. Alternatively, increased adiponectin might represent a compensatory response limiting further vascular injury [34]. To our knowledge, adiponectin levels have not been investigated in patients with JIA. In our study, we did not find statistically significant differences in levels of adiponectin between patients and controls, but adiponectin was inversely independently correlated with AIx@75 in patients with JIA, indicating that lower levels of adiponectin might be related to arterial stiffening already at an early stage of JIA.

In our study, there was no significant difference in cfPWV between the patients and the control subjects. These findings could be due to the fact that we studied newly diagnosed patients with JIA, whereas aortic stiffening may occur at later stages of the disease. Consistent with this hypothesis, a recent study has shown that patients with JIA with long-term active disease have increased aortic stiffness compared with control subjects [35].

The limitations of our study are the use of a historical control group, the gender difference and the absence of patients from all the JIA subtypes (although the two subgroups presented are the most frequent ones in every study group of patients with JIA), which limits extrapolation of the risk of vascular dysfunction to all patients with JIA. At the same time, we present a detailed, complex overview of different markers of inflammation, oxidative stress and arterial structuralfunctional properties, which describes the stage of development of subclinical atherosclerosis in these patients. Another limitation of our study is the use of generalized transfer function, which has been validated only in adults. 


\section{Conclusions}

Our study shows that patients with JIA have signs of alterations in arterial structural properties already at the time of diagnosis of the disease. Independent inverse association between the atherosclerosis-related biomarker adiponectin and arterial stiffness suggests that hypoadiponectemia may influence vascular function in these patients. Further investigations are needed to clarify the risk of vascular dysfunction during the disease course and to identify children who are at increased risk of early development of atherosclerosis in adulthood.

\begin{abstract}
Abbreviations
ADIPO: Adiponectin; ADMA: Asymmetric dimethylarginine;

Alx: Augmentation index; Alx@75: Augmentation index values adjusted to a heart rate of 75 beats/min; aPWV: Aortic pulse wave velocity; BP: Blood pressure; cfPWV: Carotid-femoral pulse wave velocity; Cl: Confidence interval; CV: Coefficient of variation; CVD: Cardiovascular disease; ELISA: Enzyme-linked immunosorbent assay; HDL: High-density lipoprotein; IMT: Intima-media thickness; JIA: Juvenile idiopathic arthritis; LDL: Low-density lipoprotein; MAP: Mean arterial pressure; MPO: Myeloperoxidase; RA: Rheumatoid arthritis; RF: Rheumatoid factor; SDS: Standard deviation score.
\end{abstract}

\section{Competing interests}

The authors declare that they have no competing interests.

\section{Authors' contributions}

$\mathrm{Jl}$ and CP were involved in the design and conduct of the study and drafted the manuscript. MZagura conducted the haemodynamic measurements, assessment of carotid intima-media thickness and revised the manuscript. ES conducted the haemodynamic measurements, assessment of carotid intima-media thickness and helped to revise the manuscript. $\mathrm{KH}$ did the investigations with controls and revised the manuscript. KZ was involved in biochemical investigations and helped to revise the manuscript. AP did the biochemical analyses and revised the manuscript. JK was involved in the design of the study and revised the manuscript. MZilmer and VT were involved in the design of the study and helped to revise the manuscript. All authors read and approved the final manuscript.

\section{Acknowledgements}

This study was funded by Estonian Science Foundation Grant No. 8750, 9094, Institutional Research Funding No IUT20-42 from the Estonian Ministry of Education and Science and by the European Union through the European Regional Development Fund (RARBSTIPP). Black Sea Trade and Investment Promotion Program is not correct.

\section{Author details}

1Department of Paediatrics, Faculty of Medicine, University of Tartu, Lunini 6, Tartu 51014, Estonia. ${ }^{2}$ Children's Clinic, Tartu University Hospital, Tartu, Estonia. ${ }^{3}$ Department of Biochemistry, Institute of Biomedicine and Translational Medicine, The Centre of Excellence for Translational Medicine, Faculty of Medicine, University of Tartu, Tartu, Estonia. ${ }^{4}$ Department of Cardiology, Faculty of Medicine, University of Tartu, Tartu, Estonia. ${ }^{5}$ Tallinn Children's Hospital, Tallinn, Estonia. ${ }^{6}$ Department of Vascular Surgery, Tartu University Hospital, Tartu, Estonia.

Received: 12 November 2014 Accepted: 26 June 2015 Published online: 16 July 2015

\section{References}

1. Al-Matar MJ, Petty RE, Tucker LB, Malleson PN, Schroeder ML, Cabral DA. The early pattern of joint involvement predicts disease progression in children with oligoarticular (pauciarticular) juvenile rheumatoid arthritis. Arthritis Rheum. 2002;46:2708-15.

2. Selvaag AM, Lien G, Sørskaar D, Vinje O, Førre $\varnothing$, Flatø B. Early disease course and predictors of disability in juvenile rheumatoid arthritis and juvenile spondyloarthropathy: a 3 year prospective study. J Rheumatol. 2005;32:1122-30.

3. Minden $K$, Niewerth $M$, Listing J, Biedermann T, Bollow M, Schöntube $M$, et al. Long-term outcome in patients with juvenile idiopathic arthritis. Arthritis Rheum. 2002:46:2392-401.

4. Flatø B, Lien G, Smerdel A, Vinje O, Dale K, Johnston V, et al. Prognostic factors in juvenile rheumatoid arthritis: a case-control study revealing early predictors and outcome after 14.9 years. J Rheumatol. 2003;30:386-93.

5. Nordal E, Zak M, Aalto K, Berntson L, Fasth A, Herlin T, et al. Ongoing disease activity and changing categories in a long-term Nordic cohort study of juvenile idiopathic arthritis. Arthritis Rheum. 2011;63:2809-18.

6. Klebanoff SJ. Myeloperoxidase: friend and foe. J Leukoc Biol. 2005;77:598-625.

7. Kwaśny-Krochin B, Głuszko P, Undas A. Plasma asymmetric dimethylarginine in active rheumatoid arthritis: links with oxidative stress and inflammation. Pol Arch Med Wewn. 2012;122:270-6.

8. Schäffler A, Ehling A, Neumann E, Herfarth H, Tarner I, Schölmerich J, et al. Adipocytokines in synovial fluid. JAMA. 2003;290:1709-10.

9. Pruunsild C, Heilman K, Zilmer K, Uibo K, Liivamägi H, Talvik T, et al. Plasma level of myeloperoxidase in children with juvenile idiopathic arthritis (a pilot study). Cent Eur J Med. 2010;5:36-40.

10. Ross R. Atherosclerosis - an inflammatory disease. N Engl J Med. 1999;340:115-26.

11. Libby P. Inflammation in atherosclerosis. Nature. 2002;420:868-74.

12. Wolfe F, Mitchell DM, Sibley JT, Fries JF, Bloch DA, Williams CA, et al. The mortality of rheumatoid arthritis. Arthritis Rheum. 1994;37:481-94.

13. del Rincón ID, Williams K, Stern MP, Freeman GL, Escalante A. High incidence of cardiovascular events in a rheumatoid arthritis cohort not explained by traditional cardiac risk factors. Arthritis Rheum. 2001:44:2737-45.

14. Aviña-Zubieta JA, Choi HK, Sadatsafavi M, Etminan M, Esdaile JM, Lacaille D Risk of cardiovascular mortality in patients with rheumatoid arthritis: a meta-analysis of observational studies. Arthritis Rheum. 2008;59:1690-7.

15. Mäki-Petäjä KM, Hall FC, Booth AD, Wallace SM. Yasmin, Bearcroft PW, et al. Rheumatoid arthritis is associated with increased aortic pulse-wave velocity, which is reduced by anti-tumor necrosis factor-a therapy. Circulation. 2006;114:1185-92.

16. Mäki-Petäjä KM, Elkhawad M, Cheriyan J, Joshi FR, Ostör AJ, Hall FC, et al. Anti-tumor necrosis factor-a therapy reduces aortic inflammation and stiffness in patients with rheumatoid arthritis. Circulation. 2012;126:2473-80.

17. Kavey RE, Allada V, Daniels SR, Hayman LL, McCrindle BW, Newburger JW, et al. Cardiovascular risk reduction in high-risk pediatric patients: a scientific statement from the American Heart Association Expert Panel on Population and Prevention Science; the Councils on Cardiovascular Disease in the Young, Epidemiology and Prevention, Nutrition, Physical Activity and Metabolism, High Blood Pressure Research, Cardiovascular Nursing, and the Kidney in Heart Disease; and the Interdisciplinary Working Group on Quality of Care and Outcomes Research: endorsed by the American Academy of Pediatrics. Circulation. 2006;114:2710-38.

18. Vlahos AP, Theocharis $P$, Bechlioulis A, Naka KK, Vakalis K, Papamichael ND, et al. Changes in vascular function and structure in juvenile idiopathic arthritis. Arthritis Care Res. 2011;63:1736-44.

19. Breda L, Di Marzio D, Giannini C, Gaspari S, Nozzi M, Scarinci A, et al. Relationship between inflammatory markers, oxidant-antioxidant status and intima-media thickness in prepubertal children with juvenile idiopathic arthritis. Clin Res Cardiol. 2013;102:63-71.

20. Satija M, Yadav TP, Sachdev N, Chhabra A, Jahan A, Dewan V. Endothelia function, arterial wall mechanics and intima media thickness in juvenile idiopathic arthritis. Clin Exp Rheumatol. 2014;32:432-9.

21. Heilman K, Zilmer M, Zilmer K, Kool P, Tillmann V. Elevated plasma adiponectin and decreased plasma homocysteine and asymmetric dimethylarginine in children with type 1 diabetes. Scand J Clin Lab Invest. 2009:69:85-91.

22. Petty RE, Southwood TR, Manners P, Baum J, Glass DN, Goldenberg J, et al. International League of Associations for Rheumatology classification of juvenile idiopathic arthritis: second revision, Edmonton, 2001. J Rheumatol. 2004;31:390-2

23. Sharman JE, Lim R, Qasem AM, Coombes JS, Burgess MI, Franco J, et al. Validation of a generalized transfer function to noninvasively derive central blood pressure during exercise. Hypertension. 2006;47:1203-8. 
24. Wilkinson IB, Fuchs SA, Jansen IM, Spratt JC, Murray GD, Cockcroft JR, et al. Reproducibility of pulse wave velocity and augmentation index measured by pulse wave analysis. J Hypertens. 1998;16:2079-84.

25. Jourdan C, Wühl E, Litwin M, Fahr K, Trelewicz J, Jobs K, et al. Normative values for intima-media thickness and distensibility of large arteries in healthy adolescents. J Hypertens. 2005:23:1707-15.

26. Dupont WD, Plummer Jr WD. Power and sample size calculations: a review and computer program. Control Clin Trials. 1990;11:116-28.

27. Stamp LK, Khalilova I, Tarr JM, Senthilmohan R, Turner R, Haigh RC, et al. Myeloperoxidase and oxidative stress in rheumatoid arthritis. Rheumatology (Oxford). 2012;51:1796-803.

28. Nicholls SJ, Hazen SL. Myeloperoxidase and cardiovascular disease Arterioscler Thromb Vasc Biol. 2005;25:1102-11.

29. Wang W, Jian Z, Guo J, Ning X. Increased levels of serum myeloperoxidase in patients with active rheumatoid arthritis. Life Sci. 2014;117:19-23.

30. Kals J, Kampus P, Kals M, Teesalu R, Zilmer K, Pulges A, et al. Arterial elasticity is associated with endothelial vasodilatory function and asymmetric dimethylarginine level in healthy subjects. Scand J Clin Lab Invest. 2007:67:536-44.

31. Ito A, Tsao PS, Adimoolam S, Kimoto M, Ogawa T, Cooke JP. Novel mechanism for endothelial dysfunction: dysregulation of dimethylarginine dimethylaminohydrolase. Circulation. 1999:99:3092-5.

32. Tain YL, Huang LT. Asymmetric dimethylarginine: clinical applications in pediatric medicine. J Formos Med Assoc. 2011;110:70-7.

33. Chow WS, Cheung BM, Tso AW, Xu A, Wat NM, Fong CH, et al. Hypoadiponectinemia as a predictor for the development of hypertension: a 5-year prospective study. Hypertension. 2007;49:1455-61.

34. Schnabel R, Messow CM, Lubos E, Espinola-Klein C, Rupprecht HJ, Bickel C, et al. Association of adiponectin with adverse outcome in coronary artery disease patients: results from the AtheroGene study. Eur Heart J. 2008:29:649-57.

35. Aulie HA, Selvaag AM, Günther A, Lilleby $V$, Molberg O, Hartmann A, et al. Arterial haemodynamics and coronary artery calcification in adult patients with juvenile idiopathic arthritis. Ann Rheum Dis. In press. 10.1136/ annrheumdis-2013-204804

\section{Submit your next manuscript to BioMed Central and take full advantage of:}

- Convenient online submission

- Thorough peer review

- No space constraints or color figure charges

- Immediate publication on acceptance

- Inclusion in PubMed, CAS, Scopus and Google Scholar

- Research which is freely available for redistribution 